
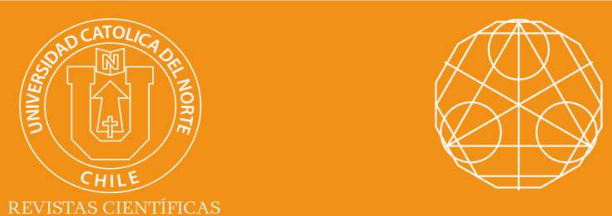

\title{
On a new difference sequence space
}

\section{P. Baliarsingh ${ }^{1}$ (D) orcid.org/0000-0002-5618-0413 \\ L. Nayak ${ }^{2}$ (1) orcid.org/0000-0003-2745-8962 \\ P. Beuria ${ }^{3}$ (1) orcid.org/0000-0002-0892-8781}

Gangadhar Meher University, Dept. of Mathematics,Sambalpur, OR, India.

1⿴ pb.math10@gmail.com

Kalinga Institute of Industrial Technology, Dept. of Mathematics, School of Applied Sci, Patia, OR, India.

2『laxmipriyamath@gmail.com ; ${ }^{3}$ beuriaprasant@gmail.com

\section{Received: May 2019 | Accepted: March 2020}

\section{Abstract:}

In the present note, we define a new difference sequence $D x=$ $(D x)_{n}$ with the help of difference operator $D$ as

$$
(D x)_{n}=\sum_{k=0}^{n}(-1)^{k}\left(\begin{array}{l}
n \\
k
\end{array}\right) x_{k}, n=0,1,2,3 \ldots
$$

Also, we discuss some interesting properties of the proposed difference operator D.

Keywords: Difference operators; Cesàro operator C(1, 1); Abel transformation; Eigenvalues.

MSC (2010): 46A45, 46A35, 46B45.

\section{Cite this article as (IEEE citation style):}

P. Baliarsingh, L. Nayak, and P. Beuria, "On a new difference sequence space", Proyecciones (Antofagasta, On line), vol. 39, no. 3 , pp. 581-589, Jun. 2020, doi: 10.22199/issn.0717-6279-202003-0035.

Article copyright: (C) 2020 P. Baliarsingh, L. Nayak and P. Beuria. This is an open access article distributed under the terms of the Creative Commons Licence, which permits unrestricted use and distribution provided the original author and source are credited.

(cc) BY 


\section{Introduction, Preliminaries and Definitions}

By $\mathbf{R}$ and $\mathbf{N}$, we denote the sets of all real numbers and nonnegative integers, respectively. Let $X$ and $Y$ be two subspaces of $w$, the space of all real valued sequences. Then we define a matrix mapping $A: X \rightarrow Y$, as

$$
(A x)_{n}:=\sum_{k} a_{n k} x_{k}, \quad(n \in \mathbf{N}) .
$$

In fact, for a sequence $x=\left(x_{k}\right) \in X, A x$ is called the $A$-transform of $x$ provided the series in (1.1) converges for each $n \in \mathbf{N}$. Moreover, the matrix $A=\left(a_{n k}\right),(n, k \in \mathbf{N})$ is also regarded as a linear operator. Initially, Kızmaz [11] introduced the idea of difference sequence spaces via difference operator $\Delta$, defined by

$$
(\Delta x)_{k}=x_{k}-x_{k+1},(k \in \mathbf{N}) .
$$

Later on, the idea has been generalized to the case of integral order $m$ by Et and Çolak [9] using the operator $\Delta^{m}$ and

$$
\left(\Delta^{m} x\right)_{k}=\sum_{i=0}^{m}(-1)^{i}\left(\begin{array}{c}
m \\
i
\end{array}\right) x_{k+i},(k \in \mathbf{N}) .
$$

Recently, Baliarsingh [3] (see also $[4,5,6]$ ) generalized the above sequence by introducing fractional difference operator $\Delta^{\alpha}$ as

$$
\left(\Delta^{\alpha} x\right)_{k}=\sum_{i=0}^{\infty}(-1)^{i} \frac{\Gamma(\alpha+1)}{i ! \Gamma(\alpha-i+1)} x_{k+i},(k \in \mathbf{N}) .
$$

Motivated by the definitions given above, we define a new difference sequence via the difference operator $D$ as

$$
(D x)_{n}=\sum_{k=0}^{n}(-1)^{k}\left(\begin{array}{c}
n \\
n-k
\end{array}\right) x_{k}=\sum_{k=0}^{n}(-1)^{k}\left(\begin{array}{l}
n \\
k
\end{array}\right) x_{k}, \quad\left(n \in \mathbf{N}_{0}=\mathbf{N} \cup\{0\}\right)
$$

Eventually, the operator $D$ is being expressed as a lower triangular matrix $D=\left(d_{n k}\right)$, where 


$$
d_{n k}= \begin{cases}(-1)^{k}\left(\begin{array}{l}
n \\
k
\end{array}\right), & (k \leq n) \\
0, & \text { (otherwise) }\end{cases}
$$

Conventionally, one may also write

$$
D=\left(\begin{array}{cccccc}
1 & 0 & 0 & 0 & 0 & \ldots \\
1 & -1 & 0 & 0 & 0 & \ldots \\
1 & -2 & 1 & 0 & 0 & \ldots \\
1 & -3 & 3 & -1 & 0 & \ldots \\
1 & -4 & 6 & -4 & 1 & \ldots \\
\vdots & \vdots & \vdots & \vdots & \vdots & \ddots
\end{array}\right)
$$

Now, we illustrate some examples of proposed difference sequence:

Let us consider a divergence sequence $x=\left(x_{k}\right)$ with $x_{k}=k$. Then the corresponding difference sequence is convergent, i.e.,

$$
(D x)_{n}=\sum_{k=0}^{n}(-1)^{k}\left(\begin{array}{l}
n \\
k
\end{array}\right) k=-n \sum_{k=1}^{n}(-1)^{k}\left(\begin{array}{l}
n-1 \\
k-1
\end{array}\right)= \begin{cases}0, & (\text { if } n>1 \text { or } n=0), \\
-1, & (\text { if } n=1) .\end{cases}
$$

If $x=e$, i.e, $x_{k}=1$ for all $k$, then the corresponding difference sequence is

$$
(D e)_{n}=\sum_{k=0}^{n}(-1)^{k}\left(\begin{array}{l}
n \\
k
\end{array}\right)= \begin{cases}0, & (\text { if } n \neq 0), \\
1, & (\text { if } n=0) .\end{cases}
$$

In fact, for most of the cases the new operators generated on various sequence spaces can be derived from respective limiting conditions of the triangular matrix $A$. The summation operator $S$ (see [8]) derived from $n$-th partial sum of the sequence $x$ is defined by $S=\left(s_{n k}\right)$, where

$$
s_{n k}= \begin{cases}1, & (k \leq n) \\ 0, & \text { (otherwise) } .\end{cases}
$$

The well known Cesàro operator $C(1,1)$ of order one is defined by $C(1,1)=\left(c_{n k}\right)($ see $[12])$, where

$$
c_{n k}= \begin{cases}\frac{1}{n+1}, & (k \leq n) \\ 0, & \text { (otherwise) }\end{cases}
$$

The backward difference operator $\Delta^{(r)}$ of order $r$ is defined by $\Delta^{(r)}=\left(\delta_{n k}^{(r)}\right)$ (see [1]), where

$$
\delta_{n k}^{(r)}= \begin{cases}(-1)^{n-k}\left(\begin{array}{c}
r \\
n-k
\end{array}\right), & (k \leq n) \\
0, & \text { (otherwise) }\end{cases}
$$


The inverse difference operator $\Delta^{(-r)}$ is being calculated as

$$
\delta_{n k}^{(-r)}= \begin{cases}(-1)^{n-k}\left(\begin{array}{c}
-r \\
n-k
\end{array}\right), & (k \leq n) \\
0, & \text { (otherwise) }\end{cases}
$$

Similarly, difference operator associated with five tuple band matrix $B(r, s, t, u, v)=\left(b_{n k}\right)$ is defined by

$$
b_{n k}= \begin{cases}r, & (k=n) \\ s, & (k=n-1) \\ t, & (k=n-2) \\ u, & (k=n-3) \\ v, & (k=n-4) \\ 0, & \text { (otherwise) }\end{cases}
$$

where $r, s, t, u, v \in \mathbf{R}$ with the condition that $r \neq 0$. In particular, the operator $B(r, s, t, u, v)$ reduces to the difference operator $B(r, s, t, u)$ studied by Baliarsingh and Dutta [7] for $v=0$, and $B(r, s)$ studied by Altay and Başar [2] for $t=u=v=0$. Also, for $u=v=0$, it reduces to the difference operator $B(r, s, t)$, studied by Furkan et al. [10].

\section{Main results}

In this section, we discuss certain results on the difference operator $D$.

Theorem 1. The operator $D$ is a linear and unbounded operator over the field $\mathbf{R}$. In fact, $\left\|D_{n}\right\|=2^{n}$, where $n$ is the order of the matrix $D_{n}$ and $\|A\|$ is the supremum over $\ell_{1}$ norms of the rows of the matrix $A$.

Proof. Proof is simple, hence omitted.

Theorem 2. (i) The matrix $D_{n}$ has its own inverse, i.e., $D_{n}=D_{n}^{-1}$.

(ii) The eigenvalues of the matrix $D_{n}$ are either +1 or -1 .

(iii) The determinant of the matrix $D_{n}$ has the absolute value 1 . 
Proof. (i) An infinite matrix $A$ is said to have its own inverse if $A=A^{-1}$, equivalently, one may also take $A^{2}=I$, where $I$ is the infinite Identity matrix which is also regarded as an identity operator. Now, for $x=\left(x_{k}\right) \in$ $c$, consider

$$
\begin{aligned}
\left(D^{2} x\right)_{n} & =D\left((D x)_{n}\right)=D\left(\sum_{k=0}^{n}(-1)^{k}\left(\begin{array}{c}
n \\
k
\end{array}\right) x_{k}\right) \\
& =D\left(x_{0}-n x_{1}+\frac{n(n-1)}{2 !} x_{n-2} \ldots+(-1)^{n} x_{n}\right) \\
& =x_{0}-n\left(x_{0}-x_{1}\right)+\frac{n(n-1)}{2 !}\left(x_{0}-2 x_{1}+x_{2}\right) \ldots \\
& +(-1)^{n}\left(x_{0}-n x_{1}+\frac{n(n-1)}{2 !} x_{n-2} \ldots+(-1)^{n} x_{n}\right) \\
& =\left(1-n+\frac{n(n-1)}{2 !} \ldots+(-1)^{n}\right) x_{0}+\left(n-2 \cdot \frac{n(n-1)}{2 !} \ldots\right. \\
& +n(-1)^{n} x_{1}+\ldots-(n-n) x_{n-1}+x_{n} \\
& =x_{n}
\end{aligned}
$$

Therefore, we conclude that $D^{2}=I$, as a result, $D_{n}=D_{n}^{-1}$.

The proof of (ii) is direct consequences of the following facts.

The operator $D_{\lambda}=D-\lambda I$, is represented by

$$
D_{\lambda}=\left(\begin{array}{cccccc}
1-\lambda & 0 & 0 & 0 & 0 & \ldots \\
1 & -1-\lambda & 0 & 0 & 0 & \ldots \\
1 & -2 & 1-\lambda & 0 & 0 & \ldots \\
1 & -3 & 3 & -1-\lambda & 0 & \ldots \\
1 & -4 & 6 & -4 & 1-\lambda & \ldots \\
\vdots & \vdots & \vdots & \vdots & \vdots & \ddots
\end{array}\right) .
$$

By straight forward calculations, the inverse matrix $D_{\lambda}^{-1}=\left(b_{n k}\right)$ can be calculated as

$$
b_{n k}= \begin{cases}\frac{1}{(-1)^{k}-\lambda}, & (k=n) \\
\frac{(-1)^{k}\left(\begin{array}{c}
n \\
n-k
\end{array}\right)}{1-\lambda^{2}}, & (k \leq n-1) \\
0, & (k>n) .\end{cases}
$$

The operator $D_{\lambda}^{-1}$ in an unbounded operator, which shows that the spectral radius of the operator $D$ is infinite. The proof of (iii) follows from (ii).

Theorem 3. (i) If $\Delta^{(r)}$ is the backward difference matrix of order $r$, then $D \Delta^{(r)} D=\Delta^{(-r)}$, where $\Delta^{(-r)}$ represents the inverse matrix of $\Delta^{(r)}$. 
(ii) If $C(1,1)$ is the Cesàro matrix of order one, then $D C(1,1) D=C(1,1)_{d}$, where $C(1,1)_{d}$ represents a diagonal matrix with the diagonal elements $1 /(n+1)$ for all $n \in \mathbf{N}$.

(iii) If $B(r, s, t, u, v)$ is the fifth order band matrix, then $D B(r, s, t, u, v) D=$ $B$, where $B=\left(b_{n k}\right)$ and

$$
b_{n k}= \begin{cases}r, & (k=n) \\ -s+(n-k-1) t-\frac{(n-k-1)(n-k-2)}{2} u+\frac{(n-k-1)(n-k-2)(n-k-3)}{6} v, & (0 \leq k<n) \\ 0, & (k>n),\end{cases}
$$

Proof. (i) Suppose $r$ is a positive integer. Then the backward difference sequence of order $r$ is given by

$$
\left(\Delta^{(r)} x\right)_{n}=\sum_{k=0}^{n}(-1)^{k}\left(\begin{array}{l}
r \\
k
\end{array}\right) x_{n-k},(n \in \mathbf{N})
$$

and the inverse transform of the difference of the sequence defined above is given by

$$
\left(\Delta^{-r} x\right)_{n}=\sum_{k=0}^{n}(-1)^{k} \frac{(r)_{k}}{k !} x_{n-k},(n \in \mathbf{N})
$$

where $(\alpha)_{k}$ denotes the Pochhammer symbol or shifted factorial of a real number $\alpha$ which is being defined using Euler gamma function as

$(\alpha)_{k}=\left\{\begin{array}{lr}1, & (\alpha=0 \quad \text { or } \quad k=0) \\ \frac{\Gamma(\alpha+k)}{\Gamma(\alpha)}=\alpha(\alpha+1)(\alpha+2) \ldots(\alpha+k-1), & (k \in \mathbf{N})\end{array}\right.$

Now, consider a sequence $x=\left(x_{n}\right) \in c$ and taking transform $D \Delta^{(r)} D$ on the sequence $x$, we have

$$
\begin{aligned}
& \mathrm{D}\left(\Delta^{(r)}(D x)\right)_{n}= \\
& D {\left[(-1)^{n} x_{n}+(-1)^{n-1}\left(\begin{array}{c}
n+r \\
1
\end{array}\right) x_{n-1}+(-1)^{n-2}\left(\begin{array}{c}
n+r \\
2
\end{array}\right) x_{n-2}+\ldots+\left(\begin{array}{c}
n+r \\
n
\end{array}\right) x_{0}\right] } \\
&=(-1)^{n}\left[(-1)^{n}\left(\begin{array}{c}
n \\
0
\end{array}\right) x_{n}+(-1)^{n-1}\left(\begin{array}{c}
n \\
1
\end{array}\right) x_{n-1}+\ldots+\left(\begin{array}{c}
n \\
2
\end{array}\right) x_{2}-\left(\begin{array}{c}
n \\
n-1
\end{array}\right) x_{1}+\left(\begin{array}{c}
n \\
n
\end{array}\right) x_{0}\right]+ \\
&(-1)^{n-1}\left(\begin{array}{c}
n+r \\
1
\end{array}\right)\left[(-1)^{n-1}\left(\begin{array}{c}
n-1 \\
0
\end{array}\right) x_{n-1}+(-1)^{n-2}\left(\begin{array}{c}
n-1 \\
1
\end{array}\right) x_{n-2}+\ldots-\left(\begin{array}{c}
n-1 \\
n-2
\end{array}\right) x_{1}+\left(\begin{array}{c}
n-1 \\
n-1
\end{array}\right) x_{0}\right]+
\end{aligned}
$$


$\vdots$

$+\left(\begin{array}{c}n+r \\ n\end{array}\right) x_{0}$

$=x_{n}+\left[-\left(\begin{array}{c}n+r \\ 0\end{array}\right)\left(\begin{array}{c}n \\ 1\end{array}\right)+\left(\begin{array}{c}n+r \\ 1\end{array}\right)\left(\begin{array}{c}n-1 \\ 0\end{array}\right)\right] x_{n-1}+\left[\sum_{i=0}^{2}(-1)^{2-i}\left(\begin{array}{c}n+r \\ i\end{array}\right)\left(\begin{array}{c}n-i \\ 2-i\end{array}\right)\right] x_{n-2}+$ $\ldots+$ $\left[\sum_{i=0}^{n}(-1)^{n-i}\left(\begin{array}{c}n+r \\ i\end{array}\right)\right] x_{0}$

$=x_{n}+(r) x_{n-1}+\left(\frac{r(r+1)}{2}\right) x_{n-2}+\ldots+\left(\frac{r(r+1) \ldots(r+n-1)}{n !}\right) x_{0}$

$=\left(\Delta^{-r} x\right)_{n}$

(ii) Applying Cesàro mean $C(1,1)$ of order one to the $D$-transform of the sequence $x=\left(x_{n}\right)$, it is seen that

$$
\mathrm{C}(1,1)(\mathrm{Dx})_{n}
$$

$=\frac{1}{n+1} \sum_{k=0}^{n}\left[(-1)^{k}\left(\begin{array}{c}k \\ 0\end{array}\right) x_{k}+(-1)^{k-1}\left(\begin{array}{c}k \\ 1\end{array}\right) x_{k-1}+\ldots+\left(\begin{array}{c}k \\ k\end{array}\right) x_{0}\right]$

$=\frac{1}{n+1}\left[x_{0}-x_{1}+x_{0}+x_{2}-2 x_{1}+x_{0}+\ldots(-1)^{n}\left(\begin{array}{c}n \\ 0\end{array}\right) x_{n}+(-1)^{n-1}\left(\begin{array}{l}n \\ 1\end{array}\right) x_{n-1}+\ldots+x_{0}\right]$

$=\frac{(-1)^{n}}{n+1} x_{n}+\left[\frac{(-1)^{n-1}}{n+1}\left(\begin{array}{c}n-1 \\ 0\end{array}\right)+\frac{(-1)^{n-1}}{n+1}\left(\begin{array}{c}n \\ 1\end{array}\right)\right] x_{n-1}+\ldots-\frac{1}{n+1}[1+2+\ldots+n] x_{1}+x_{0}$

$=\left[\sum_{i=0}^{n} \frac{(-1)^{n-i}}{n+1-i}\left(\begin{array}{c}n \\ i\end{array}\right)\right] x_{n-i}$.

Similarly, performing the difference operator $D$ in the above sequence, one can obtain that

$$
\begin{aligned}
& \mathrm{D}(\mathrm{C}(1,1)(\mathrm{D}(\mathrm{x})))_{n} \\
= & \sum_{k=0}^{n}(-1)^{k}\left(\begin{array}{c}
n \\
k
\end{array}\right)\left[\sum_{i=0}^{k} \frac{(-1)^{k-i}}{k+1-i}\left(\begin{array}{c}
k \\
i
\end{array}\right) x_{k-i}\right] \\
= & x_{0}-n\left[-\frac{x_{1}}{2}+x_{0}\right]+\frac{n(n-1)}{2}\left[\frac{x_{2}}{3}-x_{1}+x_{0}\right]+\ldots+(-1)^{n}\left[\sum_{i=0}^{n} \frac{(-1)^{n-i}}{n+1-i}\left(\begin{array}{c}
n \\
i
\end{array}\right) x_{n-i}\right] \\
= & \frac{x_{n}}{n+1}+\left[\sum_{i=0}^{1} \frac{(-1)^{1-i}}{n}\left(\begin{array}{c}
n \\
1-i
\end{array}\right)\left(\begin{array}{c}
n-1+i \\
i
\end{array}\right)\right] x_{n-1}+\left[\sum_{i=0}^{2} \frac{(-1)^{2-i}}{n-1}\left(\begin{array}{c}
n \\
2-i
\end{array}\right)\left(\begin{array}{c}
n-2+i \\
i
\end{array}\right)\right] x_{n-2}+ \\
+ & \ldots+\left[\sum_{i=0}^{n}(-1)^{n-i}\left(\begin{array}{c}
n \\
n-i
\end{array}\right)\right] x_{0} \\
= & \frac{x_{n}}{n+1} \text { (iii) The proof is quite similar to that of (ii), }
\end{aligned}
$$

Theorem 4. The Abel type transformation for the operator $D$ is given by

$$
\sum_{k=0}^{n} x_{k}(D y)_{k}=\sum_{j=0}^{n}(-1)^{j} y_{j} \sum_{k=j}^{n}\left(\begin{array}{l}
k \\
j
\end{array}\right) x_{k} .
$$

Proof. Taking the LHS, we have

$$
\begin{aligned}
& \sum_{k=0}^{n} x_{k}(D y)_{k}=\sum_{k=0}^{n} x_{k} \sum_{i=0}^{k}(-1)^{i}\left(\begin{array}{c}
k \\
i
\end{array}\right) y_{i} \\
= & \sum_{k=0}^{n} x_{k}\left[y_{0}-k y_{1}+\frac{k(k+1)}{2} y_{2}+\ldots+(-1)^{k} y_{k}\right] \\
= & x_{0} y_{0}+x_{1}\left(y_{0}-y_{1}\right)+x_{2}\left(y_{0}-2 y_{1}+y_{2}\right)+x_{3}\left(y_{0}-3 y_{1}+3 y_{2}-y_{1}\right)+\ldots+
\end{aligned}
$$


$x_{n}\left(y_{0}-n y_{1}+\frac{n(n+1)}{2} y_{2}+\ldots+(-1)^{n} y_{n}\right)$

$=y_{0}\left(x_{0}+x_{1}+\ldots+x_{n}\right)-y_{1}\left(x_{1}+2 x_{2}+\ldots+n x_{n}\right)+y_{2}\left(x_{2}+3 x_{3}+\ldots+\frac{n(n+1)}{2} x_{n}\right)$

$y_{n}(-1)^{n} x_{n}$

$=\sum_{j=0}^{n}(-1)^{j} y_{j} \sum_{k=j}^{n}\left(\begin{array}{c}k \\ j\end{array}\right) x_{k}$. This completes the proof.

\section{References}

[1] Z. U. Ahmad, M. Mursaleen, "Köthe-Toeplitz duals of some new sequence spaces and their matrix maps", Publications de L'Institut Mathématique, vol. 42, no. 56, pp. 57-61, 1987. [On line]. Available: https://bit.ly/2AXioWz

[2] B. Altay and F. Bașar, "On the fine spectrum of the difference operator $\Delta$ on $c_{0}$ and $c^{\prime \prime}$, Information sciences, vol. 168 , no. $1-4$, pp. $217-$ 224, Dec. 2004, doi: 10.1016/j.ins.2004.02.007

[3] P. Baliarsingh, "Some new difference sequence spaces of fractional order and their dual spaces", Applied mathematics and computation, vol. 219, no. 18, pp. 9737-9742, May 2013, doi: 10.1016/j.amc.2013.03.073

[4] P. Baliarsingh and S. Dutta, "A unifying approach to the difference operators and their applications", Boletim da Sociedade Paranaense de Matemática, vol. 33, no. 1, p. 49, 2013., doi: 10.5269/bspm.v33i1.19884

[5] P. Baliarsingh and S. Dutta, "On the classes of fractional order difference sequence spaces and their matrix transformations", Applied mathematics and computation, vol. 250, pp. 665-674, Jan. 2015, doi: 10.1016/j.amc.2014.10.121

[6] P. Baliarsingh, "On a fractional difference operator", Alexandria engineering journal, vol. 55, no. 2, pp. 1811-1816, Jun. 2016, doi: 10.1016/j.aej.2016.03.037

[7] P. Baliarsingh and S. Dutta, "On certain Toeplitz matrices via difference operator and their applications", Afrika matematika, vol. 27, no. 5-6, pp. 781-793, Aug. 2015, doi:10.1007/s13370-015-0374-z

[8] B. Choudhary, S.K. Mishra, "On Köthe-Toeplitz duals of certain sequence spaces and their matrix transformations", Indian journal of pure and applied mathematics, vol. 24, no. 5, pp. 291301, 1993. [On line]. Available: https://bit.ly/3bVmNWz

[9] M. Et and R. Çolak, "On some generalized difference sequence spaces", Soochow journal of mathematics, vol. 21, no. 4, pp. 377386, Oct. 1995. [On line]. Available: https://bit.ly/2AWvT8V 
[10] H. Bilgic and H. Furkan, "On the fine spectrum of the generalized difference operator $B(r, s)$ over the sequence spaces $\ell_{\mathrm{p}}$ and $\mathrm{b} v_{p},(1<\mathrm{p}<\infty)$ ", Nonlinear analysis: theory, methods \& applications, vol. 68, no. 3, pp. 499506, Feb. 2008, doi: 10.1016/j.na.2006.11.015

[11] H. Kizmaz, "On certain sequence spaces", Canadian mathematical bulletin, vol. 24, no. 2, pp. 169-176, Jun. 1981, doi: 10.4153/CMB-1981-027-5

[12] P-N. Ng and P-Y. Lee, "Cesàro sequence spaces of non-absolute type", Commentationes mathematicae, vol. 20, no. 2, pp. 429-433, 1978. [On line]. Available: https://bit.ly/2ZvWi7z 